\title{
TYPE II EXTINCTION PROFILE OF MAXIMAL SOLUTIONS TO THE RICCI FLOW IN $\mathbb{R}^{2}$
}

\author{
PANAGIOTA DASKALOPOULOS* AND NATASA SESUM
}

\begin{abstract}
We consider the initial value problem $u_{t}=\Delta \log u, u(x, 0)=$ $u_{0}(x) \geq 0$ in $\mathbb{R}^{2}$, corresponding to the Ricci flow, namely conformal evolution of the metric $u\left(d x_{1}^{2}+d x_{2}^{2}\right)$ by Ricci curvature. It is well known that the maximal (complete) solution $u$ vanishes identically after time $T=\frac{1}{4 \pi} \int_{\mathbb{R}^{2}} u_{0}$. Assuming that $u_{0}$ is compactly supported we describe precisely the Type II vanishing behavior of $u$ at time $T$ : we show the existence of an inner region with exponentially fast vanishing profile, which is, up to proper scaling, a soliton cigar solution, and the existence of an outer region of persistence of a logarithmic cusp. This is the only Type II singularity which has been shown to exist, so far, in the Ricci Flow in any dimension. It recovers rigorously formal asymptotics derived by J.R. King [28].
\end{abstract}

\section{INTRODUCTION}

We consider the Cauchy problem

$$
\begin{cases}u_{t}=\Delta \log u & \text { in } \mathbb{R}^{2} \times(0, T) \\ u(x, 0)=u_{0}(x) & x \in \mathbb{R}^{2}\end{cases}
$$

for the logarithmic fast diffusion equation in $\mathbb{R}^{2}$, with $T>0$ and initial data $u_{0}$ non-negative, bounded and compactly supported.

It has been observed by S. Angenent and L. Wu [30, 31] that equation (1.1) represents the evolution of the conformally equivalent metric $g_{i j}=u d x_{i} d x_{j}$ under the Ricci Flow

$$
\frac{\partial g_{i j}}{\partial t}=-2 R_{i j}
$$

which evolves $g_{i j}$ by its Ricci curvature. The equivalence easily follows from the observation that the conformal metric $g_{i j}=u I_{i j}$ has scalar curvature $R=$ $-(\Delta \log u) / u$ and in two dimensions $R_{i j}=\frac{1}{2} R g_{i j}$.

* : Partially supported by the NSF grants DMS-01-02252, DMS-03-54639 and the EPSRC in the UK. 
Equation (1.1) arises also in physical applications, as a model for long Van-derWals interactions in thin films of a fluid spreading on a solid surface, if certain nonlinear fourth order effects are neglected, see [10, 5, 6].

It is shown in [1] that given an initial data $u_{0} \geq 0$ with $\int_{\mathbb{R}^{2}} u_{0} d x<\infty$ and a constant $\gamma \geq 2$, there exists a solution $u_{\gamma}$ of (1.1) with

$$
\int_{\mathbb{R}^{2}} u_{\lambda}(x, t) d x=\int_{\mathbb{R}^{2}} u_{0} d x-2 \pi \gamma t .
$$

The solution $u_{\gamma}$ exists up to the exact time $T=T_{\gamma}$, which is determined in terms of the initial area and $\gamma$ by $T_{\gamma}=\frac{1}{2 \pi \gamma} \int_{\mathbb{R}} u_{0} d x$.

We restrict our attention to maximal solutions $u$ of (1.1), corresponding to the value $\gamma=2$ in (1.3), which vanish at time

$$
T=\frac{1}{4 \pi} \int_{\mathbb{R}^{2}} u_{0}(x) d x .
$$

It is shown in [11] and 29] that if $u_{0}$ is compactly supported, then the maximal solution $u$ which extincts at time $T$ satisfies the asymptotic behavior

$$
u(x, t)=\frac{2 t}{|x|^{2} \log ^{2}|x|}(1+o(1)), \quad \text { as } \quad|x| \rightarrow \infty, \quad 0 \leq t<T .
$$

This bound, of course, deteriorates as $t \rightarrow T$. Geometrically (1.5) corresponds to the condition that the conformal metric is complete. The manifold can be visualized as a surface of finite area with an unbounded cusp.

J.R. King 28 has formally analyzed the extinction behavior of maximal solutions $u$ of (1.1), as $t \rightarrow T^{-}$. His analysis, for radially symmetric and compactly supported initial data, suggests the existence of two regions of different behavior: in the outer region $(T-t) \log r>T$ the "logarithmic cusp" exact solution $2 t /|x|^{2} \log ^{2}|x|$ of equation $u_{t}=\Delta \log u$ persists. However, in the inner region $(T-t) \log r \leq T$ the solution vanishes exponentially fast and approaches, after an appropriate change of variables, one of the soliton solutions $U$ of equation $U_{\tau}=\Delta \log U$ on $-\infty<\tau<\infty$ given by $U(x, \tau)=1 /\left(\lambda|x|^{2}+e^{4 \lambda \tau}\right)$, with $\tau=1 /(T-t)$ and $\lambda=T / 2$.

This behavior was established rigorously in the radially symmetric case by the first author and M. del Pino in 12. The precise asymptotics of the Ricci flow neckpinch in the compact case, on $S^{n}$, has been established by Knopf and Angenent in 2 .

Our goal in this paper is to remove the assumption of radial symmetry and establish the vanishing behavior of maximal solutions of (1.1) for any non-negative compactly supported initial data. 
TYPE II EXTINCTION PROFILE OF MAXIMAL SOLUTIONS TO THE RICCI FLOW IN $\mathbb{R}^{2} 3$

To state the inner region behavior in a precise manner, we perform the change of variables

$$
\bar{u}(x, \tau)=\tau^{2} u(x, t), \quad \tau=\frac{1}{T-t}
$$

and

$$
\tilde{u}(y, \tau)=\alpha(\tau) \bar{u}\left(\alpha(\tau)^{1 / 2} y, \tau\right)
$$

with

$$
\alpha(\tau)=[\bar{u}(0, \tau)]^{-1}=\left[(T-t)^{-2} u(0, t)\right]^{-1}
$$

so that $\tilde{u}(0, \tau)=1$.

A direct computation shows that the rescaled solution $\tilde{u}$ satisfies the equation

$$
\tilde{u}_{\tau}=\Delta \log \tilde{u}+\frac{\alpha^{\prime}(\tau)}{2 \alpha(\tau)} \nabla(y \cdot \tilde{u})+\frac{2 \tilde{u}}{\tau} .
$$

Then, following result holds:

Theorem 1.1. (Inner behavior) Assume that the initial data $u_{0}$ is non-negative, bounded and compactly supported. Then,

$$
\lim _{\tau \rightarrow \infty} \frac{\alpha^{\prime}(\tau)}{2 \alpha(\tau)}=T
$$

and the rescaled solution $\tilde{u}$ defined by (1.6) - (1.8) converges, uniformly on compact subsets of $\mathbb{R}^{2}$, to the solution

$$
U(x)=\frac{1}{\frac{T}{2}|y|^{2}+1}
$$

of the steady state equation

$$
\Delta \log U+T \cdot \nabla(y \cdot U)=0 .
$$

Since for any maximal solution $T=(1 / 4 \pi) \int_{\mathbb{R}^{2}} u_{0}(x) d x$, this theorem shows, in particular, that the limit of the rescaled solution is uniquely determined by the area of the initial data. The uniqueness of the limit has not previously shown in 12 even under the assumption of radial symmetry.

To describe the vanishing behavior of $u$ in the outer region we first perform the cylindrical change of variables

$$
v(\zeta, \theta, t)=r^{2} u(r, \theta, t), \quad \zeta=\log r
$$


with $(r, \theta)$ denoting the polar coordinates. Equation $u_{t}=\Delta \log u$ in cylindrical coordinates takes the form

$$
v_{t}=\Delta_{c} \log v
$$

with $\Delta_{c}$ denoting the Laplacian in cylindrical coordinates defined as

$$
\Delta_{c} \log v=(\log v)_{\zeta \zeta}+(\log v)_{\theta \theta} .
$$

We then perform a further scaling setting

$$
\tilde{v}(\xi, \theta, \tau)=\tau^{2} v(\tau \xi, \theta, t), \quad \tau=\frac{1}{T-t} .
$$

A direct computation shows that $\tilde{v}$ satisfies the equation

$$
\tau \tilde{v}_{\tau}=\frac{1}{\tau}(\log \tilde{v})_{\xi \xi}+\tau(\log \tilde{v})_{\theta \theta}+\xi \tilde{v}_{\xi}+2 \tilde{v} .
$$

The extinction behavior of $u$ (or equivalently of $v$ ) in the outer region $\xi \geq T$, is described in the following result.

Theorem 1.2. (Outer behavior). Assume that the initial data $u_{0}$ is non-negative, bounded and compactly supported. Then, the rescaled solution $\tilde{v}$ defined by (1.13) converges, as $\tau \rightarrow \infty$, to the $\theta$-independent steady state solution $V(\xi)$ of equation (1.14) given by

$$
V(\xi)= \begin{cases}\frac{2 T}{\xi^{2}}, & \xi>T \\ 0, & \xi<T .\end{cases}
$$

Moreover, the convergence is uniform on the set $\left(-\infty, \xi^{-}\right] \times[0,2 \pi]$, for any $-\infty<$ $\xi^{-}<T$, and on compact subsets of $(T,+\infty) \times[0,2 \pi]$.

Under the assumption of radial symmetry this result follows from the work of the first author and del Pino [12].

The proof of Theorems 1.1 and 1.2 rely on sharp estimates on the geometric width $W$ and on the maximum curvature $R_{\max }$ of maximal solutions near their extinction time $T$ derived in 13 by the first author and R. Hamilton. In particular, it is found in [13] that the maximum curvature is proportional to $1 /(T-t)^{2}$, which does not go along with the natural scaling of the problem which would entail blow-up of order $1 /(T-t)$. One says that the vanishing behavior is of type II. The proof also makes an extensive use of the Harnack estimate on the curvature $R=-\Delta \log u / u$ shown by Hamilton and Yau in [18. Although the result in 18 is shown only for 
TYPE II EXTINCTION PROFILE OF MAXIMAL SOLUTIONS TO THE RICCI FLOW IN $\mathbb{R}^{2} 5$

a compact surface evolving by the Ricci flow, we shall observe in section 2 that the result remains valid in our case as well. Finally, let us remark that the proof of the inner-region behavior is based on the classification of eternal complete solutions of the 2-dimensional Ricci flow, recently shown by the authors in [14].

\section{Preliminaries}

In this section we will collect some preliminary results which will be used throughout the rest of the paper. For the convenience of the reader, we start with a brief description of the geometric estimates in [13] on which the proofs of Theorems 1.1 and 1.2 rely upon.

2.1. Geometric Estimates. In 13 the first author and R. Hamilton established upper and lower bounds on the geometric width $W(t)$ of the maximal solution $u$ of (1.1) and on the maximum curvature $R_{\max }(t)=\max _{x \in \mathbb{R}^{2}} R(x, t)$, with $R=-(\Delta \log u) / u$.

Let $F: \mathbb{R}^{2} \rightarrow[0, \infty)$ denote a proper function $F$, such that $F^{-1}(a)$ is compact for every $a \in[0, \infty)$. The width of $F$ is defined to be the supremum of the lengths of the level curves of $F$, namely $w(F)=\sup _{c} L\{F=c\}$. The width $w$ of the metric $g$, as introduced in [13, is defined to be the infimum

$$
w(g)=\inf _{F} w(F) .
$$

The estimates in [13] depend on the time to collapse $T-t$. However, they do not scale in the usual way. More precisely:

Theorem 2.1 (Daskalopoulos and Hamilton [13]). There exist positive constants $c$ and $C$ for which

$$
c(T-t) \leq W(t) \leq C(T-t)
$$

and

$$
\frac{c}{(T-t)^{2}} \leq R_{\max }(t) \leq \frac{C}{(T-t)^{2}}
$$

for all $0<t<T$.

2.2. The Hamilton-Yau Harnack estimate. In 18 Hamilton and Yau established a Harnack estimate on the curvature $R$ of a compact surface evolving by the Ricci flow, in the case where the curvature $R$ changes sign. Since the proof in 18 uses only local quantities, the result and its proof can be carried over to the complete, non-compact case. 
Theorem 2.2 (Hamilton and Yau [18]). For any constants $E$ and $L$ we can find positive constants $A, B, C, D$ such that for any complete solution to the Ricci flow on $\mathbb{R}^{2}$ which at the initial time $t=0$ satisfies

$$
R \geq 1-E
$$

and

$$
\frac{1}{R+E} \frac{\partial R}{\partial t}-\frac{|\nabla R|^{2}}{(R+E)^{2}} \geq-L
$$

then, for all $t \geq 0$ we have

$$
\frac{1}{R+E} \frac{\partial R}{\partial t}-\frac{|\nabla R|^{2}}{(R+E)^{2}}+F\left(\frac{|\nabla R|^{2}}{(R+E)^{2}}, R+E\right) \geq 0
$$

where

$$
F(X, Y)=A+\sqrt{2 B(X+Y)+C}+D \log Y
$$

Integrating the above estimate along paths we obtain:

Proposition 2.3. Under the assumptions of Theorem 1.1, there exist uniform constants $E>0$ and $C_{1}, C_{2}>0$ so that for every $x_{1}, x_{2} \in \mathbb{R}^{2}$ and $T / 2<t_{1}<t_{2}$ we have

$$
\frac{1}{\sqrt{R\left(x_{1}, t_{1}\right)+E}} \geq \frac{1}{\sqrt{R\left(x_{2}, t_{2}\right)+E}}-C_{1}\left(t_{2}-t_{1}\right)-C_{2} \frac{\operatorname{dist}_{t_{1}}^{2}\left(x_{1}, x_{2}\right)}{t_{2}-t_{1}} .
$$

Proof. By the Aronson-Benilán inequality $R \geq-1 / t \geq-2 / T=1-E$ for all $t \in[T / 2, T)$. Hence the estimate (2.4) and the lower curvature bound on $R$ give

$$
\begin{aligned}
\frac{\partial R}{\partial t} & \geq \frac{|\nabla R|^{2}}{R+E}-(2 A+\sqrt{C})(R+E)-\sqrt{2 B}|\nabla R| \\
& -\sqrt{2 B}(R+E) \sqrt{R+E}-D \log (R+E)(R+E) \\
& \geq \frac{|\nabla R|^{2}}{R+E}-A_{1}(R+E) \sqrt{R+E}-\frac{1}{4} \frac{|\nabla R|^{2}}{R+E} \\
& =\frac{3}{4} \frac{|\nabla R|^{2}}{R+E}-A_{1}(R+E) \sqrt{R+E} .
\end{aligned}
$$

Take any two points $x_{1}, x_{2} \in \mathbb{R}^{2}$ and $T / 2 \leq t_{1} \leq t_{2}<T$ and let $\gamma$ be a curve connecting $x_{1}$ and $x_{2}$, such that $\gamma\left(t_{1}\right)=x_{1}$ and $\gamma\left(t_{2}\right)=x_{2}$. Since

$$
\frac{d}{d t} R(\gamma(t), t)=\frac{\partial R}{\partial t}+\langle\nabla R, \dot{\gamma}\rangle
$$

using also (2.6) we find

$$
\begin{aligned}
\frac{d}{d t} R(\gamma(t), t) & \geq \frac{3}{4} \frac{|\nabla R|^{2}}{R+E}-A_{1}(R+E) \sqrt{R+E}-C|\dot{\gamma}|^{2}(R+E)-\frac{1}{4} \frac{|\nabla R|^{2}}{R+E} \\
& \geq-A_{3}(R+E)^{3 / 2}\left(1+|\dot{\gamma}|^{2}\right) .
\end{aligned}
$$


TYPE II EXTINCTION PROFILE OF MAXIMAL SOLUTIONS TO THE RICCI FLOW IN $\mathbb{R}^{2} 7$

Integrating the previous equation along the path $\gamma$, gives

$$
\frac{1}{\sqrt{R\left(x_{1}, t_{1}\right)+E}} \geq \frac{1}{\sqrt{R\left(x_{2}, t_{2}\right)+E}}-C\left(t_{2}-t_{1}\right)-\int_{t_{1}}^{t_{2}}|\dot{\gamma}|_{g(t)}^{2} d t
$$

Due to the bound $R \geq 1-E$ we have for $t \geq s$

$$
|\dot{\gamma}|_{g(t)}^{2} \leq(1-E) e^{s t}|\dot{\gamma}|_{g(s)}^{2}
$$

and if we choose the curve $\gamma$ to be the minimal geodesic with respect to metric $g\left(t_{1}\right)$ connecting $x_{1}$ and $x_{2}$ we obtain

$$
\frac{1}{\sqrt{R\left(x_{1}, t_{1}\right)+E}} \geq \frac{1}{\sqrt{R\left(x_{2}, t_{2}\right)+E}}-C_{1}\left(t_{2}-t_{1}\right)-C_{2} \frac{\operatorname{dist}_{t_{1}}^{2}\left(x_{1}, x_{2}\right)}{t_{2}-t_{1}}
$$

as desired.

2.3. Monotonicity of Solutions. Our solution $u(x, t)$ to (1.1) has compactly supported initial data. The classical argument based on reflection, due to Alexandrov and Serrin, proves that such solutions enjoy the following monotonicity in the radial direction:

Lemma 2.4. Under the assumptions of Therorem 1.1 if $\operatorname{supp} u_{0}(\cdot) \subset B_{\rho}(0)$, then

$$
u(x, t) \geq u(y, t)
$$

for all $t \in(0, T)$ and every pair of points $x, y \in \mathbb{R}^{2}$ such that $|y| \geq|x|+\rho$.

The proof of Lemma 2.4 is the same as the proof of Proposition 2.1 in [4. For the reader's convenience we will briefly sketch it.

Sketch of the proof. Assume that $\rho=1$. By the comparison principle for maximal solutions it easily follows that if $K=\operatorname{supp} u(\cdot, 0)$ and $K \subset\left\{x \in \mathbb{R}^{2}: x_{2}>0\right\}$, then $u\left(x_{1}, x_{2}, t\right) \geq u\left(x_{1},-x_{2}, t\right)$ for $x_{1} \in \mathbb{R}^{+}$and $t \in[0, T)$. Fix $x^{0} \in B_{1}$ and $x^{1} \in \partial B_{1+\delta}$ for $\delta>0$. Let $\Pi$ be a hyperplane of points in $\mathbb{R}^{2}$ which are equidistant from $x^{0}$ and $x^{1}$. Then, it easily follows

$$
\operatorname{dist}(\Pi,\{0\}) \geq 1
$$

which implies $x^{0}$ and $\operatorname{supp} u(\cdot, 0)$ are in the same half-space with respect to $\Pi$. Since $x^{1}$ is the reflection of $x^{0}$ in $\Pi$, it follows $u\left(x^{0}, t\right) \geq u\left(x^{1}, t\right)$. We can now let $\delta \rightarrow 0$ to get the claim. 
Notice that due to (1.5), for every $t \in(0, T)$ we can define $x_{t}$ to be such that $u\left(x_{t}, t\right)=\max _{\mathbb{R}^{2}} u(\cdot, t)$. An easy consequence of Lemma 2.4 is the following result about $\left\{x_{t}\right\}_{t \in(0, T)}$.

Corollary 2.5. For every $t \in(0, T), x_{t} \in B_{2 \rho}(0)$.

\section{Inner Region Convergence}

This section is devoted to the proof of the inner region convergence, Theorem 1.1 stated in the Introduction. We assume, throughout this section, that $u$ is a smooth, maximal solution of (1.1) with compactly supported initial data $u_{0}$ and $u$ a maximal solution that vanishes at time

$$
T=\frac{1}{4 \pi} \int_{\mathbb{R}^{2}} u_{0} d x .
$$

3.1. Scaling and convergence. We introduce a new scaling on the solution $u$ namely

$$
\bar{u}(x, \tau)=\tau^{2} u(x, t), \quad \tau=\frac{1}{T-t}, \quad \tau \in(1 / T, \infty) .
$$

Then $\bar{u}$ satisfies the equation

$$
\bar{u}_{\tau}=\Delta \log \bar{u}+\frac{2 \bar{u}}{\tau}, \quad \text { on } \quad 1 / T \leq \tau<\infty .
$$

Notice that under this transformation, $\bar{R}:=-\Delta \log \bar{u} / \bar{u}$ satisfies the estimate

$$
\bar{R}_{\max }(\tau) \leq C
$$

for some constant $C<\infty$. This is a direct consequence of Theorem [2.1] since $\bar{R}_{\max }(\tau)=(T-t)^{2} R_{\max }(t)$.

For an increasing sequence $\tau_{k} \rightarrow \infty$ we set

$$
\bar{u}_{k}(y, \tau)=\alpha_{k} \bar{u}\left(\alpha_{k}^{1 / 2} y, \tau+\tau_{k}\right), \quad(y, \tau) \in \mathbb{R}^{2} \times\left(-\tau_{k}+1 / T, \infty\right)
$$

where

$$
\alpha_{k}=\left[\bar{u}\left(0, \tau_{k}\right)\right]^{-1}
$$

so that $\bar{u}_{k}(0,0)=1$, for all $k$. Then, $\bar{u}_{k}$ satisfies the equation

$$
\bar{u}_{\tau}=\Delta \log \bar{u}+\frac{2 \bar{u}}{\tau+\tau_{k}} .
$$

Let

$$
\bar{R}_{k}:=-\frac{\Delta \log \bar{u}_{k}}{\bar{u}_{k}} .
$$


TYPE II EXTINCTION PROFILE OF MAXIMAL SOLUTIONS TO THE RICCI FLOW IN $\mathbb{R}^{2} 9$

Then, by (3.3), we have

$$
\max _{y \in \mathbb{R}^{2}} \bar{R}_{k}(y, \tau) \leq C, \quad-\tau_{k}+1 / T<\tau<+\infty .
$$

We will also derive a global bound from bellow on $\bar{R}_{k}$. The Aronson-Benilán inequality $u_{t} \leq u / t$, on $0 \leq t<T$, gives the bound $R(x, t) \geq-1 / t$ on $0 \leq t<T$. In particular, $R(x, t) \geq-C$ on $T / 2 \leq t<T$, which in the new time variable $\tau=1 /(T-t)$ implies the bound

$$
\bar{R}(x, \tau) \geq-\frac{C}{\tau^{2}}, \quad 2 / T<\tau<\infty .
$$

Hence

$$
\bar{R}_{k}(y, \tau) \geq-\frac{C}{\left(\tau+\tau_{k}\right)^{2}}, \quad-\tau_{k}+2 / T<\tau<+\infty .
$$

Combining the above inequalities we get

$$
-\frac{C}{\left(\tau+\tau_{k}\right)^{2}} \leq \bar{R}_{k}(y, \tau) \leq C, \quad \forall(y, \tau) \in \mathbb{R}^{2} \times\left(-\tau_{k}+2 / T,+\infty\right) .
$$

Based on the above estimates we will now show the following convergence result.

Lemma 3.1. For each sequence $\tau_{k} \rightarrow \infty$, there exists a subsequence $\tau_{k_{l}}$ of $\tau_{k}$, for which the rescaled solution $\bar{u}_{\tau_{k_{l}}}$ defined by (3.4) converges, uniformly on compact subsets of $\mathbb{R}^{2} \times \mathbb{R}$, to an eternal solution $U$ of equation $U_{\tau}=\Delta \log U$ on $\mathbb{R}^{2} \times \mathbb{R}$ with uniformly bounded curvature and uniformly bounded width. Moreover, the convergence is in $C^{\infty}(K)$, for any $K \subset \mathbb{R}^{2} \times \mathbb{R}$ compact.

Proof. Denote by $x_{k}=x_{t_{k}}$ the maximum point of $u\left(\cdot, t_{k}\right)$. First, instead of rescaling our solution by $\alpha_{k}$ we can rescale it by $\beta_{k}=\left[\bar{u}\left(x_{k}, \tau_{k}\right)\right]^{-1}$, that is, consider

$$
\tilde{u}_{k}(y, \tau)=\beta_{k} \bar{u}\left(\beta_{k}^{1 / 2} y, \tau+\tau_{k}\right), \quad \tau \in\left(-\tau_{k}+1 / T, \infty\right) .
$$

For $y_{k}=\beta_{k}^{-1 / 2} x_{k}$ we have $\tilde{u}_{k}\left(y_{k}, 0\right)=1$ and $\tilde{u}_{k}(\cdot, 0) \leq 1$ since $x_{k}$ is the maximum point of $u\left(\cdot, t_{k}\right)$. Notice that $\left|y_{k}\right| \leq 2 \rho \beta_{k}^{-1 / 2}$, because $x_{k} \in B_{2 \rho}$, by Corollary 2.5. Since $\tilde{u}_{k}$ satisfies (3.5), standard arguments imply that $\tilde{u}_{k}$ is uniformly bounded from above and below away from zero on any compact subset of $\mathbb{R}^{2} \times \mathbb{R}$. In particular, there are uniform constants $C_{1}>0$ and $C_{2}<\infty$ so that

$$
C_{1} \leq \frac{\alpha_{k}}{\beta_{k}} \leq C_{2} .
$$

Let $K \subset \mathbb{R}^{2}$ be a compact set. By (3.8), for every compact set $K$ there is a compact set $K^{\prime}$ so that for all $y \in K$ we have $y\left(\frac{\alpha_{k}}{\beta_{k}}\right)^{1 / 2} \in K^{\prime}$, for all $k$. Also, by the previous 
estimates we have

$$
C_{1}\left(K^{\prime}\right) \leq \frac{\bar{u}\left(\beta_{k}^{1 / 2} z, \tau_{k}+\tau\right)}{\bar{u}\left(x_{k}, \tau_{k}\right)}=\tilde{u}_{k}(z, \tau) \leq C_{2}\left(K^{\prime}\right)
$$

for all $z \in K^{\prime}$ and $\tau$ belonging to a compact subset of $(-\infty, \infty)$. Therefore, using (3.8) and remembering that $\alpha_{k}=\left[\bar{u}\left(0, \tau_{k}\right)\right]^{-1}$ we find

$$
\bar{u}_{k}(y, \tau)=\frac{\bar{u}\left(\alpha_{k}^{1 / 2} y, \tau+\tau_{k}\right)}{\bar{u}\left(0, \tau_{k}\right)} \leq \frac{1}{C_{1}} \frac{\bar{u}\left(\beta_{k}^{1 / 2}\left[\left(\frac{\alpha_{k}}{\beta_{k}}\right)^{1 / 2} y\right], \tau_{k}+\tau\right)}{\bar{u}\left(x_{k}, \tau_{k}\right)} \leq \frac{C_{2}\left(K^{\prime}\right)}{C_{1}}=C_{2}(K) .
$$

Similarly,

$$
C_{1}(K)=\frac{C_{1}\left(K^{\prime}\right)}{C_{2}} \leq \frac{\bar{u}\left(\alpha_{k}^{1 / 2} y, \tau_{k}+\tau\right)}{\bar{u}\left(0, \tau_{k}\right)}=\bar{u}_{k}\left(y, \tau_{k}\right)
$$

for $y \in K$ and $\tau$ belonging to a compact set. Hence, by the classical regularity theory the sequence $\left\{\bar{u}_{k}\right\}$ is equicontinuous on compact subsets of $\mathbb{R}^{2} \times \mathbb{R}$. It follows that there exists a subsequence $\tau_{k_{l}}$ of $\tau_{k}$ such that $\bar{u}_{k_{l}} \rightarrow U$ on compact subsets of $\mathbb{R}^{2} \times \mathbb{R}$, where $U$ is an eternal solution of equation

$$
U_{\tau}=\Delta \log U, \quad \text { on } \mathbb{R}^{2} \times \mathbb{R}
$$

with infinite area $\int_{\mathbb{R}^{2}} U(y, \tau) d y=\infty\left(\right.$ since $\left.\int_{\mathbb{R}^{2}} \bar{u}_{k}(y, \tau)=2\left(\tau+\tau_{k}\right)\right)$. In addition the classical regularity theory of quasilinear parabolic equations implies that $\left\{u_{k_{l}}\right\}$ can be chosen so that $u_{k_{l}} \rightarrow U$ in $C^{\infty}(K)$, for any compact set $K \subset \mathbb{R}^{2} \times \mathbb{R}$, with $U(0,0)=1$.

It then follows that $\bar{R}_{k_{l}} \rightarrow \bar{R}:=-(\Delta \log U) / U$. Taking the limit $k_{l} \rightarrow \infty$ on both sides of (3.7) we obtain the bounds

$$
0 \leq \bar{R} \leq C, \quad \text { on } \quad \mathbb{R}^{2} \times \mathbb{R}
$$

Finally, to show that $U$ has uniformly bounded width, we take the limit $k_{l} \rightarrow \infty$ in (2.1).

As direct consequence of Lemma 3.1 and the classification result of eternal solutions to the complete Ricci flow on $\mathbb{R}^{2}$, recently showed in [14], we obtain the following convergence result.

Theorem 3.2. For each sequence $\tau_{k} \rightarrow \infty$, there exists a subsequence $\tau_{k_{l}}$ of $\tau_{k}$ and numbers $\lambda, \bar{\lambda}>0$ for which the rescaled solution $\bar{u}_{\tau_{k_{l}}}$ defined by (3.4) converges, uniformly on compact subsets of $\mathbb{R}^{2} \times \mathbb{R}$, to the soliton solution $U$ of the Ricci Flow given by

$$
U(y, \tau)=\frac{1}{\lambda|y|^{2}+e^{4 \bar{\lambda} \tau}}
$$


Moreover, the convergence is in $C^{\infty}(K)$, for any $K \subset \mathbb{R}^{2} \times \mathbb{R}$, compact.

Proof. ¿From Lemma $3.1 \bar{u}_{\tau_{k_{l}}} \rightarrow U$, where $U$ is an eternal solution of $U_{t}=\Delta \log U$, on $\mathbb{R}^{2} \times \mathbb{R}$, with uniformly bounded width, such that $\sup _{\mathbb{R}^{2}} R(\cdot, t) \leq C(t)<\infty$ for every $t \in(-\infty, \infty)$. The main result in [14] shows that the limiting solution $U$ is a soliton of the form $U(x, \tau)=\frac{2}{\beta\left(\left|x-x_{0}\right|^{2}+\delta e^{2 \beta t}\right)}$, with $\beta>0, \delta>0$, which under the condition $U(0,0)=1$ takes the form $U(x, \tau)=\frac{1}{\lambda\left|x-x_{0}\right|^{2}+e^{4 \lambda \tau}}$, with $\lambda, \bar{\lambda}>0$.

It remains to show that the limit $U(\cdot, \tau)$ is rotationally symmetric around the origin, that is, $x_{0}=0$. This will follow from Lemma 2.4 and Lemma 3.1. Notice that $\lim _{k \rightarrow \infty} \alpha_{k}=\infty$. Since $\bar{u}_{k}\left(\cdot, \tau_{k}\right)$ converges uniformly on compact subsets of $\mathbb{R}^{2} \times \mathbb{R}$ to a cigar soliton $U(y, 0)$, we have that

$$
\begin{aligned}
\bar{u}\left(0, \tau_{k}\right) & =\tau_{k}^{2} u\left(0, t_{k}\right) \approx \frac{1}{\lambda\left|x_{0}\right|^{2}+e^{4 \bar{\lambda} \tau_{k}}} \\
& \leq e^{-4 \bar{\lambda} \tau_{k}} \rightarrow 0,
\end{aligned}
$$

as $k \rightarrow \infty$ and therefore $\lim _{k \rightarrow \infty} \alpha_{k}=\infty$. Lets us express $\bar{u}=\bar{u}(r, \theta, \tau)$ in polar coordinates. For every $r>0$ there is $k_{0}$ so that $\alpha_{k}^{1 / 2} r>1$ for $k \geq k_{0}$. By Lemma 2.4

$$
\begin{aligned}
\min _{\theta} \bar{u}\left(\alpha_{k}^{1 / 2} r, \theta, \tau_{k}\right) & \geq \max _{\theta} \bar{u}\left(\alpha_{k}^{1 / 2} r+1, \theta, \tau_{k}\right) \\
& =\max _{\theta} \bar{u}\left(\alpha_{k}^{1 / 2}\left(r+\alpha_{k}^{-1 / 2}\right), \theta, \tau_{k}\right)
\end{aligned}
$$

which implies

$$
\min _{\theta} \bar{u}_{k}(r, \theta, 0) \geq \max _{\theta} \bar{u}_{k}\left(r+\alpha_{k}^{-1 / 2}, \theta, 0\right) .
$$

Let $k \rightarrow \infty$ to obtain

$$
\min _{\theta} U(r, \theta, 0) \geq \max _{\theta} U(r, \theta, 0)
$$

which yields the limit $U(r, \theta, 0)$ is radially symmetric with respect to the origin and therefore $x_{0}=0$, implying that $U$ is of the form (3.11).

3.2. Further behavior. We will now use the geometric properties of the rescaled solutions and their limit, to further analyze their vanishing behavior. Our analysis will be similar to that in 12, applicable to the nonradial case as well. However, the uniqueness of the limit along sequences $\tau_{k} \rightarrow \infty$ which will be shown in Theorem 3.9] is an improvement of the results in [12, even in the radial case.

We begin by observing that rescaling back in the original $(x, t)$ variables, Theorem 3.2 gives the following asymptotic behavior of the maximal solution $u$ of (1.1). 
Lemma 3.3. Assuming that along a sequence $t_{k} \rightarrow T$, the sequence $\bar{u}_{k}$ defined by (3.4) with $\tau_{k}=\left(T-t_{k}\right)^{-1}$ converges to the soliton solution $U_{\lambda}$, on compact subsets of $\mathbb{R}^{2} \times \mathbb{R}$, then along the sequence $t_{k}$ the solution $u(x, t)$ of (1.1) satisfies the asymptotics

$$
u\left(x, t_{k}\right) \approx \frac{\left(T-t_{k}\right)^{2}}{\lambda|x|^{2}+\alpha_{k}}, \quad \text { on } \quad|x| \leq \alpha_{k}^{1 / 2} M
$$

for all $M>0$. In addition, the curvature $R\left(0, t_{k}\right)=-\Delta \log u\left(0, t_{k}\right) / u\left(0, t_{k}\right)$ satisfies

$$
\lim _{t_{k} \rightarrow T}\left(T-t_{k}\right)^{2} R\left(0, t_{k}\right)=4 \lambda .
$$

The proof of Lemma above is the same as the proof of Lemma 3.3 in 12. The following Lemma provides a sharp bound from below on the maximum curvature $4 \lambda$ of the limiting solitons.

Lemma 3.4. Under the assumptions of Theorem 1.1 the constant $\lambda$ in each limiting solution (B.11) satisfies

$$
\lambda \geq \frac{T}{2}
$$

Proof. We are going to use the estimate proven in Section 2 of [13]. It is shown there that if at time $t$ the solution $u$ of (1.1) satisfies the scalar curvature bound $R(t) \geq-2 k(t)$, then the width $W(t)$ of the metric $u(t)\left(d x_{1}^{2}+d x_{2}^{2}\right)$ (c.f. in Section 2.1 for the definition) satisfies the bound

$$
W(t) \leq \sqrt{k(t)} A(t)=4 \pi \sqrt{k(t)}(T-t) .
$$

Here $A(t)=4 \pi(T-t)$ denotes the area of the plane with respect to the conformal metric $u(t)\left(d x_{1}^{2}+d x_{2}^{2}\right)$. Introducing polar coordinates $(r, \theta)$, let

$$
\bar{U}(r, t)=\max _{\theta} u(r, \theta, t) \quad \text { and } \quad \underline{\mathrm{U}}(r, t)=\min _{\theta} u(r, \theta, t) .
$$

Then

$$
\underline{\mathrm{U}}(r, t) \leq u(r, \theta, t) \leq \bar{U}(r, t)
$$

implying the bound

$$
W(\underline{\mathrm{U}}(t)) \leq W(t) \leq 4 \pi \sqrt{k(t)}(T-t) .
$$

Observe next that the Aronson-Benilán inequality on $u$ implies the bound $R(x, t) \geq$ $-1 / t$. Hence we can take $k(t)=\frac{1}{2 t}$ in (3.14). Observing that for the radially 
TYPE II EXTINCTION PROFILE OF MAXIMAL SOLUTIONS TO THE RICCI FLOW IN $\mathbb{R}^{2} 13$

symmetric solution $\underline{\mathrm{U}}$ the width $W(\underline{\mathrm{U}})=\max _{r \geq 0} 2 \pi r \sqrt{\underline{\mathrm{U}}}(r, t)$, we conclude the pointwise estimate

$$
2 \pi r \sqrt{\underline{\mathrm{U}}}(r, t) \leq \frac{4 \pi(T-t)}{\sqrt{2 t}}, \quad r \geq 0,0<t<T .
$$

By Lemma (2.4),

$$
r \sqrt{u}(r+\rho, \theta, t) \leq r \sqrt{\underline{\mathrm{U}}}(r, t), \quad \text { for } r>0 .
$$

For a sequence $t_{k} \rightarrow T$, let $\alpha_{k}=\left[\bar{u}\left(0, \tau_{k}\right)\right]^{-1}, \tau_{k}=1 /\left(T-t_{k}\right)$, as before. Using (3.12), 3.15) and (3.16) we find

$$
\frac{r\left(T-t_{k}\right)}{\sqrt{\lambda(r+\rho)^{2}+\alpha_{k}}} \leq \frac{2\left(T-t_{k}\right)}{\sqrt{2 t_{k}}}, \quad r \leq M \alpha_{k}^{1 / 2}
$$

for any positive number $M$. Hence, when $r=M \alpha_{k}^{1 / 2}$ we obtain the estimate

$$
\frac{M \alpha_{k}^{1 / 2}}{\sqrt{\lambda\left(M+\rho \alpha_{k}^{-1 / 2}\right)^{2} \alpha_{k}+\alpha_{k}}} \leq \frac{2}{\sqrt{2 t_{k}}}
$$

or

$$
\frac{M}{\sqrt{\lambda\left(M+\rho \alpha_{k}^{-1 / 2}\right)^{2}+1}} \leq \frac{2}{\sqrt{2 t_{k}}} .
$$

Letting $t_{k} \rightarrow T$ and taking squares on both sides, we obtain

$$
\frac{1}{\lambda+1 / M^{2}} \leq \frac{2}{T} \text {. }
$$

Since $M>0$ is an arbitrary number, we finally conclude $\lambda \geq T / 2$, as desired.

We will next provide a bound on the behavior of $\alpha(\tau)=\tau^{2} \bar{u}(0, \tau)$, as $\tau \rightarrow \infty$. In particular, we will prove (1.10). We begin by a simple consequence of Lemma 3.4

Lemma 3.5. Under the assumptions of Theorem 3.2 we have

$$
\liminf _{\tau \rightarrow \infty} \frac{\alpha^{\prime}(\tau)}{\alpha(\tau)} \geq 4 \lambda_{0}
$$

with $\lambda_{0}=T / 2$.

The proof of Lemma 3.5 is the same as the proof of Lemma 3.5 in [12.

Corollary 3.6. Under the hypotheses of Theorem 3.2 we have

$$
\alpha(\tau) \geq e^{2 T \tau+o(\tau)}, \quad \text { as } \quad \tau \rightarrow \infty .
$$

The next Proposition will be crucial in establishing the outer region behavior of $u$. 
Proposition 3.7. Under the hypotheses of Theorem 1.1, we have

$$
\lim _{\tau \rightarrow \infty} \frac{\log \alpha(\tau)}{\tau}=2 T
$$

Proof. See Proposition 3.7 in [12.

A consequence of Lemma 3.3 and Proposition 3.7 is the following result, which will be used in the next section.

Corollary 3.8. Under the assumptions of Lemma 3.1 the rescaled solution $\tilde{v}$ defined by (1.13) satisfies

$$
\lim _{\tau \rightarrow \infty} \tilde{v}(\xi, \theta, \tau)=0, \quad \text { uniformly on }(\xi, \theta) \in\left(-\infty, \xi^{-}\right] \times[0,2 \pi]
$$

for all $\xi^{-}<T$.

So far we have showed that $\bar{\lambda}=\lim _{\tau \rightarrow \infty} \frac{\log \alpha(\tau)}{\tau}=T / 2$ and that $\lambda \geq T / 2$. In the next theorem we will show that actually $\lambda=T / 2$. Theorem 3.9 is an improvement of the results in [12, since it leads to the uniqueness of a cigar soliton limit.

Theorem 3.9. $\lim _{\tau \rightarrow \infty} \bar{R}(0, \tau)=2 T$.

We will first prove the following lemma.

Lemma 3.10. For every $\beta>1$ and for every sequence $\tau_{i} \rightarrow \infty$ there is a sequence $s_{i} \in\left(\tau_{i}, \beta \tau_{i}\right)$ such that $\lim _{i \rightarrow \infty} \bar{R}\left(0, s_{i}\right)=2 T$.

Proof. By definition

$$
(\log \alpha(\tau))_{\tau}=\bar{R}(0, \tau)-\frac{2}{\tau}
$$

Therefore

$$
\log \alpha\left(\beta \tau_{i}\right)-\log \alpha\left(\tau_{i}\right)=\left(\bar{R}\left(0, s_{i}\right)-\frac{2}{s_{i}}\right)(\beta-1) \tau_{i} .
$$

Since $\log \alpha(\tau)=2 T \tau+o(\tau)$, by Proposition 3.7 we conclude

$$
\left(\bar{R}\left(0, s_{i}\right)-\frac{2}{s_{i}}\right)(\beta-1)=\frac{\left(2 T \beta \tau_{i}+o\left(\beta \tau_{i}\right)\right)-\left(2 T \tau_{i}+o\left(\tau_{i}\right)\right)}{\tau_{i}}
$$

which yields

$$
\bar{R}\left(0, s_{i}\right)=2 T+\frac{2}{s_{i}}+\frac{o\left(\beta \tau_{i}\right)+o\left(\tau_{i}\right)}{\tau_{i}}
$$

readily implying the Lemma. 
Proof of Theorem 3.9. By Lemma 3.4 we have $\lambda \geq T / 2$. Assume there is a sequence $\tau_{i} \rightarrow \infty$ such that $\lim _{i \rightarrow \infty} \bar{R}\left(0, \tau_{i}\right)=4 \lambda$, where $4 \lambda=2 T+\delta$ for some $\delta>0$. We know that $\bar{R}(0, \tau) \leq \tilde{C}$ for a uniform constant $\tilde{C}$. Choose $\beta>1$ so that the following two conditions hold

$$
\frac{1}{\beta \sqrt{2 \tilde{C}}}>C(\beta-1)
$$

and

$$
2 T+\frac{\delta}{2}>2 T\left(\frac{\beta}{1-C(\beta-1) \sqrt{2 T}}\right)^{2}
$$

for some uniform constant $C$ to be chosen later. Notice that both (3.20) and (3.21) are possible by choosing $\beta>1$ suffciently close to 1 . By Lemma 3.10 find a sequence $s_{i} \in\left(\tau_{i}, \beta \tau_{i}\right)$ so that $\lim _{i \rightarrow \infty} \bar{R}\left(0, s_{i}\right)=2 T$. Let $T / 2<t<T$. Then $R(x, t) \geq-\frac{2}{T}=1-E$. Hamilton-Yau Harnack estimate (2.5), applied to $t_{i}$ (where $\left.\tau_{i}=\frac{1}{T-t_{i}}\right)$ and $\bar{t}_{i}>t_{i}\left(\right.$ where $\frac{1}{T-t_{i}}=\beta \tau_{i}$ for $\beta>1$ ), yields

$$
\frac{1}{\sqrt{\bar{R}\left(0, \tau_{i}\right)+\frac{E}{\tau_{i}^{2}}}} \geq \frac{\tau_{i}}{s_{i} \sqrt{\bar{R}\left(0, s_{i}\right)+\frac{E}{s_{i}^{2}}}}-C \frac{s_{i}-\tau_{i}}{s_{i}} .
$$

Notice that due to our choice of $\beta$ in (3.20) we have

$$
\begin{aligned}
\frac{\tau_{i}}{s_{i} \sqrt{\bar{R}\left(0, s_{i}\right)+\frac{E}{s_{i}^{2}}}} & \geq \frac{\tau_{i}}{s_{i} \sqrt{2 \tilde{C}}} \\
& \geq \frac{1}{\beta \sqrt{2 \tilde{C}}} \geq C(\beta-1) \geq C \frac{s_{i}-\tau_{i}}{s_{i}} .
\end{aligned}
$$

Therefore

$$
\begin{aligned}
\sqrt{\bar{R}\left(0, \tau_{i}\right)+\frac{E}{\tau_{i}^{2}}} & \leq \frac{1}{\frac{\tau_{i}}{s_{i} \sqrt{\bar{R}\left(0, s_{i}\right)+\frac{E}{s_{i}^{2}}}}-C \frac{s_{i}-\tau_{i}}{s_{i}}} \\
& =\frac{s_{i} \sqrt{\bar{R}\left(0, s_{i}\right)+\frac{E}{s_{i}^{2}}}}{\tau_{i}-C\left(s_{i}-\tau_{i}\right) \sqrt{\bar{R}\left(0, s_{i}\right)+\frac{E}{s_{i}^{2}}}} .
\end{aligned}
$$

Denote by $A=\sqrt{\bar{R}\left(0, s_{i}\right)+\frac{E}{s_{i}^{2}}}$. Since the function $f(x)=\frac{A x}{\tau_{i}-C A\left(x-\tau_{i}\right)}$, for $x \in$ $\left[\tau_{i}, \beta \tau_{i}\right]$ is increasing, we conclude

$$
\begin{aligned}
\sqrt{\bar{R}\left(0, \tau_{i}\right)+\frac{E}{\tau_{i}^{2}}} & \leq \frac{\beta \tau_{i} \sqrt{\bar{R}\left(0, s_{i}\right)+\frac{E}{s_{i}^{2}}}}{\tau_{i}-C\left(\beta \tau_{i}-\tau_{i}\right) \sqrt{\bar{R}\left(0, s_{i}\right)+\frac{E}{s_{i}^{2}}}} \\
& =\frac{\beta \sqrt{\bar{R}\left(0, s_{i}\right)+\frac{E}{s_{i}^{2}}}}{1-C(\beta-1) \sqrt{\bar{R}\left(0, s_{i}\right)+\frac{E}{s_{i}^{2}}}} .
\end{aligned}
$$


Letting $i \rightarrow \infty$ in (3.22) we get

$$
\sqrt{4 \lambda} \leq \frac{\beta \sqrt{2 T}}{1-C(\beta-1) \sqrt{2 T}}
$$

which implies

$$
2 T+\delta=2 T\left(\frac{\beta}{1-C(\beta-1) \sqrt{2 T}}\right)^{2}
$$

contradicting our choice of $\beta$ in 3.21).

3.3. Proof of Theorem 1.1. We finish this section with the proof of Theorem 1.1 which easily follows from the results in Sections 3.1 and 3.2 Take any sequence $\tau_{k} \rightarrow \infty$. Observe that by Theorem 3.9

$$
\lim _{k \rightarrow \infty} \frac{\alpha^{\prime}\left(\tau_{k}\right)}{\alpha\left(\tau_{k}\right)}=2 T
$$

By the definitions of $\tilde{u}$ and $\bar{u}_{k}$ (1.7) and (3.4) respectively) we have $\tilde{u}\left(y, \tau_{k}\right)=$ $\bar{u}_{k}(y, 0)$. By Theorem 3.2 we have $\bar{u}_{k} \rightarrow U_{\frac{T}{2}}$ and therefore

$$
\tilde{u}\left(y, \tau_{k}\right) \rightarrow U_{\frac{T}{2}}(y, 0)=\frac{1}{\frac{T}{2}|y|^{2}+1} .
$$

The limit $U_{\frac{T}{2}}$ does not depend on the sequence $t_{k} \rightarrow T$ and the proof of Theorem 1.1 is now complete.

\section{Outer Region Asymptotic Behavior}

We assume, throughout this section, that $u$ is a positive, smooth, maximal solution of (1.1) satisfying the assumptions of Theorem 1.2 which vanishes at time

$$
T=\frac{1}{4 \pi} \int_{\mathbb{R}^{2}} u_{0} d x
$$

As in Introduction we consider the solution $v(\zeta, \theta, t)=r^{2} u(r, \theta, t), \zeta=\log r$, of the equation (1.12) in cylindrical coordinates. We next set

$$
\bar{v}(\zeta, \theta, \tau)=\tau^{2} v(\zeta, \theta, t), \quad \tau=\frac{1}{T-t} .
$$

and

$$
\tilde{v}(\xi, \tau)=\bar{v}(\tau \xi, \tau) .
$$

The function $\tilde{v}$ satisfies the equation

$$
\tau \tilde{v}_{\tau}=\frac{1}{\tau}(\log \tilde{v})_{\xi \xi}+\tau(\log \tilde{v})_{\theta \theta}+\xi \tilde{v}_{\xi}+2 \tilde{v} .
$$

Note that the curvature $R=-\Delta_{c} \log v / v$, is given in terms of $\tilde{v}$ by

$$
R(\tau \xi, \theta, t)=-\frac{(\log \tilde{v})_{\xi \xi}(\xi, \theta, t)+\tau^{2}(\log \tilde{v})_{\theta \theta}(\xi, \theta, t)}{\tilde{v}} .
$$


TYPE II EXTINCTION PROFILE OF MAXIMAL SOLUTIONS TO THE RICCI FLOW IN $\mathbb{R}^{2} 17$

Moreover, the area of $\tilde{v}$ is constant, in particular

$$
\int_{-\infty}^{\infty} \int_{0}^{2 \pi} \tilde{v}(\xi, \theta, \tau) d \theta d \xi=4 \pi, \quad \forall \tau
$$

We shall show that, $\tilde{v}(\cdot, \tau)$ converges, as $\tau \rightarrow \infty$, to a $\theta$-independent steady state of equation (4.3), namely to a solution of the linear first order equation

$$
\xi V_{\xi}+2 V=0 .
$$

The area condition (4.5) shall imply that

$$
\int_{-\infty}^{\infty} V(\xi) d \xi=2
$$

Positive solutions of equation (4.6) are of the form

$$
V(\xi)=\frac{\eta}{\xi^{2}}
$$

where $\eta>0$ is any constant. These solutions become singular at $\xi=0$ and in particular are non-integrable at $\xi=0$, so that they do not satisfy the area condition (4.7). However, it follows from Corollary 3.8 that $V$ must vanish in the interior region $\xi<T$. We will show that while $\tilde{v}(\xi, \theta, \tau) \rightarrow 0$, as $\tau \rightarrow \infty$ on $(-\infty, T)$, we have $\tilde{v}(\xi, \theta, \tau) \geq c>0$, for $\xi>T$ and that actually $\tilde{v}(\xi, \theta, \tau) \rightarrow 2 T / \xi^{2}$, on $(T, \infty)$, as stated in Theorem 1.2

The rest of the section is devoted to the proof of Theorem 1.2 We begin by showing the following properties of the rescaled solution $\tilde{v}$.

Lemma 4.1. The rescaled solution $\tilde{v}$ given by (4.1) - (4.2) has the following properties:

i. $\tilde{v}(\cdot, \tau) \leq C$, for a constant $C$ independent of $\tau$.

ii. For any $\xi^{-}<T, \tilde{v}(\cdot, \tau) \rightarrow 0$, as $\tau \rightarrow \infty$, uniformly on $\left(-\infty, \xi^{-}\right] \times[0,2 \pi]$.

iii. Let $\xi(\tau)=(\log \alpha(\tau)) / 2 \tau$, with $\alpha(\tau)=\left[\tau^{2} u(0, t)\right]^{-1}$. Then, there exists $\tau_{0}>0$ and a constant $\eta>0$, independent of $\tau$, such that

$$
\tilde{v}(\xi, \theta, \tau) \geq \frac{\eta}{\xi^{2}}, \quad \text { on } \xi \geq \xi(\tau), \tau \geq \tau_{0} .
$$

In addition

$$
\xi(\tau)=T+o(1), \quad \text { as } \tau \rightarrow \infty .
$$

iv. $\tilde{v}(\xi, \theta, \tau)$ also satisfies the upper bound

$$
\tilde{v}(\xi, \theta, \tau) \leq \frac{C}{\xi^{2}}, \quad \text { on } \xi>0, \tau \geq \tau_{0}
$$

for some constants $C>0$ and $\tau_{0}>0$. 
Proof. (i) One can easily show using the maximum principle that $v(\zeta, \theta, t) \leq C / s^{2}$, for $s_{0}$ sufficiently large, with $C$ independent of $t$. This implies the bound $\tilde{v}(\xi, \theta, \tau) \leq$ $C / \xi^{2}$, for $\xi \tau>s_{0}$. On the other hand, by Corollary 3.8 we have $\tilde{v}(\xi, \theta, \tau) \leq C$, on $\xi<\xi^{-}<T$, with $C$ independent of $\tau$. Combining the above, the desired estimate follows.

(ii) This is shown in Corollary 3.8

(iii) We have shown in the previous section that the rescaled solution $\bar{u}(x, \tau)=$ $\tau^{2} u(x, t), \tau=1 /(T-t)$, defined by (3.1) satisfies the asymptotics $\bar{u}(x, \tau) \approx$ $1 /\left(\frac{T}{2}|x|^{2}+\alpha(\tau)\right)$, when $|x| \leq \sqrt{\alpha(\tau)}$. Hence

$$
\tilde{v}(\xi(\tau), \theta, \tau)=\bar{v}(\xi(\tau) \tau, \theta, \tau) \approx \frac{e^{2 \xi(\tau) \tau}}{\lambda e^{2 \xi(\tau) \tau}+\alpha(\tau)} \approx \frac{1}{\frac{T}{2}+1}
$$

if $\xi(\tau)=\frac{\log \alpha(\tau)}{2 \tau}$.

Observe next that (4.10) readily follows from Proposition 3.7 Hence, it remains to show $\tilde{v} \geq \eta / \xi^{2}$, for $\xi \in[\xi(\tau), \infty), \tau_{0} \leq \tau<\infty$. To this end, we will compare $\tilde{v}$ with the subsolution $V_{\eta}(\xi, \theta)=\eta / \xi^{2}$ of equation (4.3). According to our claim above, there exists a constant $\eta>0$, so that

$$
V_{\eta}(\xi(\tau), \theta)=\frac{\eta}{\xi(\tau)^{2}} \leq \tilde{v}(\xi(\tau), \theta, \tau)
$$

Moreover, by the growth condition (1.5), we can make

$$
\tilde{v}\left(\xi, \theta, \tau_{0}\right)>\frac{\eta}{\xi^{2}}, \quad \text { on } \quad \xi \geq \xi\left(\tau_{0}\right)
$$

by choosing $\tau_{0}>0$ and $\eta$ sufficiently small. By the comparison principle, (4.9) follows.

(iv) Since $u_{0}$ is compactly supported and bounded, it follows that $u_{0}(r) \leq 2 A /\left(r^{2} \log ^{2} r\right)$, on $r>1$ for some $A>0$. Since $2(t+A) /\left(r^{2} \log ^{2} r\right)$ is an exact solution of equation (1.1), it follows by the comparison principle on that $u(r, t) \leq 2(t+A) /\left(r^{2} \log ^{2} r\right)$, for $r>1$, which readily implies the desired bound on $\tilde{v}$, with $C=2(A+T)$.

Lemma 4.2. For any compact set $K \subset(T, \infty)$, there is a constant $C(K)$ for which

$$
\max _{\xi \in K}\left|\int_{0}^{2 \pi}(\log \tilde{v})_{\xi}(\xi, \theta, \tau) d \theta\right| \leq C(K), \quad \forall \tau \geq 2 / T
$$


TYPE II EXTINCTION PROFILE OF MAXIMAL SOLUTIONS TO THE RICCI FLOW IN $\mathbb{R}^{2} 19$

Proof. We integrate (4.4) in $\theta$ variable and use the bounds $R \geq-1 / t \geq-2 / T$, for $t \geq T / 2$, and $\tilde{v}(\tau \xi, t) \leq C$ shown in Lemma 4.1 to get

$$
\int_{0}^{2 \pi}(\log \tilde{v})_{\xi \xi}(\xi, \theta, \tau) d \theta \leq C
$$

for all $\tau=1 /(T-t) \geq 2 / T$. We can now proceed as in the proof of Lemma 4.2 in [12] to show (4.11).

To simplify the notation, we set

$$
R_{c}(\zeta, \theta, t)=R(r, \theta, t), \quad r=\log \zeta
$$

Lemma 4.3. For any compact set $K \subset(T, \infty)$, there is a constant $C(K)$ such that for any $\xi_{0} \in K$ and $\gamma>0$

$$
\min _{\left[\xi_{0}, \xi_{0}+\gamma \frac{\log \tau}{\tau}\right] \times[0,2 \pi]} R_{c}(\xi \tau, \theta, t) \leq \frac{C(K) \tau}{\gamma \log \tau} .
$$

Proof. Assume that for some $K$ and $\gamma, \min _{\left[\xi_{0}, \xi_{0}+\gamma \frac{\log \tau}{\tau}\right] \times[0,2 \pi]} R_{c}(\xi \tau, \theta, t) \geq \frac{M \tau}{\gamma \log \tau}$, for $M$ large. Then, it follows from (4.4) and the bound $\tilde{v} \leq C$ shown in Lemma 4.1 that for every $\xi \in\left[\xi_{0}, \xi_{0}+\gamma \frac{\log \tau}{\tau}\right]$ we have

$$
\begin{aligned}
\int_{0}^{2 \pi}(\log \tilde{v})_{\xi \xi}(\xi, \theta, \tau) d \theta & \left.=-\int_{0}^{2 \pi} R_{c}(\xi \tau, \theta, t)\right) \tilde{v}(\tau \xi, \theta, t) d \theta \\
& \leq-\frac{C}{\xi^{2}} \min _{\left[\xi_{0}, \xi_{0}+\gamma \frac{\log \tau}{\tau}\right] \times[0,2 \pi]} R_{c}(\xi \tau, \theta, t) \\
& \leq-C_{1}(K) \frac{M \tau}{\gamma \log \tau}
\end{aligned}
$$

which combined with Lemma 4.2 implies

$$
\begin{aligned}
-C(K) & \leq \int_{0}^{2 \pi}(\log \tilde{v})_{\xi}(\xi, \theta, \tau) d \theta \\
& \leq \int_{0}^{2 \pi}(\log \tilde{v})_{\xi}\left(\xi_{0}, \theta, \tau\right) d \theta-C_{1}(K) \frac{\gamma \log \tau}{\tau} \frac{M \tau}{\gamma \log \tau} \\
& =\int_{0}^{2 \pi}(\log \tilde{v})_{\xi}\left(\xi_{0}, \theta, \tau\right) d \theta-C_{1}(K) M \leq C(K)-C_{1}(K) M
\end{aligned}
$$

impossible if $M$ is chosen sufficiently large.

Proposition 4.4. For every $K \subset(T, \infty)$ compact, there is a constant $C(K)$ depending only on $K$, such that for any $\xi_{0} \in K$

$$
\max _{\left[\xi_{0}, \xi_{0}+\frac{\log \tau}{\tau}\right] \times[0,2 \pi]} R_{c}(\xi \tau, \theta, t) \leq \frac{C(K) \tau}{\log \tau} .
$$


Proof. Let $\xi_{1} \in K, \theta \in[0,2 \pi]$ and $\tau_{1}$ be arbitrary. Choose $\xi_{2}$ such that $T<\xi_{2}<$ $\min K$ and $\tau_{2}$ such that $\xi_{1} \tau_{1}=\xi_{2} \tau_{2}$. Since $\xi_{2}<\xi_{1}$, then $\tau_{2}>\tau_{1}$. Set $t_{i}=T-1 / \tau_{i}$, $i=1,2$. We next define the set $A_{\xi_{2}}=\left\{\xi: \xi_{2} \leq \xi \leq \xi_{2}+\gamma \frac{\log \tau_{2}}{\tau_{2}}\right\}$. Let $\xi_{0} \in A_{\xi_{2}}$ and $\theta_{2} \in[0,2 \pi]$ be such that

$$
R_{c}\left(\xi_{0} \tau_{2}, \theta_{2}, t_{2}\right)=\min _{(\xi, \theta) \in A_{\xi_{2}} \times[0,2 \pi]} R_{c}\left(\xi \tau_{2}, \theta, t_{2}\right)
$$

and set $x_{1}=\left(e^{\xi_{1} \tau_{1}}, \theta_{1}\right)$ and $x_{2}=\left(e^{\xi_{0} \tau_{2}}, \theta_{2}\right)$. Since $\xi_{1} \tau_{1}=\xi_{2} \tau_{2} \leq \xi_{0} \tau_{2}$, then $\left|x_{1}\right| \leq\left|x_{2}\right|$. Denoting by $\operatorname{dist}_{t_{1}}\left(x_{1}, x_{2}\right)$ the distance with respect to the metric $g_{t_{1}}=u\left(\cdot, t_{1}\right)\left(d x^{2}+d y^{2}\right)$, we have:

Claim 4.5. For any $0<\gamma<1$, there is a constant $C=C(K, \gamma)$ so that

$$
\operatorname{dist}_{t_{1}}\left(x_{1}, x_{2}\right) \leq \frac{C(K, \gamma)}{\tau_{1}^{1-\gamma}} \text {. }
$$

Proof of Claim. We have seen in the proof of Lemma 4.1 that $u(x, t) \leq \frac{C}{|x|^{2} \log ^{2}|x|}$, for all $|x| \geq 1$ and all $t \in[0, T)$. If $\sigma$ is a euclidean geodesic with respect to $g_{t_{1}}=u\left(\cdot, t_{1}\right)\left(d x^{2}+d y^{2}\right)$, connecting $x_{1}$ and $x_{2}$, this implies

$$
\begin{aligned}
\operatorname{dist}_{t_{1}}\left(x_{1}, x_{2}\right) & \leq \int_{\sigma} \sqrt{u}\left(\cdot, t_{1}\right) d \sigma \\
& \leq C \frac{\left|e^{\xi_{1} \tau_{1}}-e^{\xi_{0} \tau_{2}}\right|}{e^{\xi_{1} \tau_{1}} \xi_{1} \tau_{1}} \\
& \leq C \frac{e^{\xi_{2} \tau_{2}+\gamma \log \tau_{2}}-e^{\xi_{1} \tau_{1}}}{e^{\xi_{1} \tau_{1}} \xi_{1} \tau_{1}} \\
& \leq \frac{C}{\xi_{1} \tau_{1}}\left(e^{\gamma \log \tau_{2}}-1\right)=\frac{C \xi_{1}^{\gamma-1}}{\xi_{2}^{\gamma} \tau_{1}^{1-\gamma}}-\frac{C}{\xi_{1} \tau_{1}} \\
& \leq \frac{C \xi_{1}^{\gamma-1}}{\xi_{2}^{\gamma} \tau_{1}^{1-\gamma}} \leq \frac{A(K, \gamma)}{\tau_{1}^{1-\gamma}} .
\end{aligned}
$$

To finish the proof of the Proposition, we first apply the Harnack estimate (2.5) to obtain the inequality

$$
\frac{1}{\sqrt{R_{c}\left(\xi_{1} \tau_{1}, \theta_{1}, t_{1}\right)+E}} \geq \frac{1}{\sqrt{R_{c}\left(\xi_{0} \tau_{2}, \theta_{2}, t_{2}\right)+E}}-C\left(t_{2}-t_{1}\right)-C \frac{\operatorname{dist}_{t_{1}}^{2}\left(x_{1}, x_{2}\right)}{t_{2}-t_{1}} .
$$

By Lemma 4.3. using also that $\xi_{1} \tau_{1}=\xi_{2} \tau_{2}$ (since $\tau_{2}>\tau_{1}$, we have $\xi_{1}>\xi_{2}$ ), we get

$$
\begin{aligned}
\frac{1}{\sqrt{R_{c}\left(\xi_{1} \tau_{1}, \theta_{1}, t_{1}\right)+E}} & \geq \frac{C(K, \gamma) \sqrt{\log \tau_{2}}}{\sqrt{\tau_{2}}}-\frac{C\left(\xi_{1}-\xi_{2}\right)}{\xi_{2} \tau_{2}}-\frac{C(K, \gamma)}{\tau_{1}^{1-2 \gamma}} \frac{\tau_{2}}{\left(\tau_{2}-\tau_{1}\right)} \\
& =\frac{C(K, \gamma) \sqrt{\log \tau_{2}}}{\sqrt{\tau_{2}}}-\frac{C_{1}(K, \gamma)}{\tau_{2}}-\frac{C_{2}(K, \gamma)}{\tau_{1}^{1-2 \gamma}}
\end{aligned}
$$


TYPE II EXTINCTION PROFILE OF MAXIMAL SOLUTIONS TO THE RICCI FLOW IN $\mathbb{R}^{2} 21$

In the last inequality we used that

$$
\frac{\tau_{2}}{\left(\tau_{2}-\tau_{1}\right)}=\frac{1}{\left(1-\tau_{1} / \tau_{2}\right)}=\frac{1}{\left(1-\xi_{2} / \xi_{1}\right)}=\frac{\xi_{1}}{\left(\xi_{1}-\xi_{2}\right)}
$$

and that $\left(\xi_{1}-\xi_{2}\right) / \xi_{2}$ and $\xi_{1} /\left(\xi_{1}-\xi_{2}\right)$ depend only on the set $K$.

Take $\gamma=\frac{1}{4}$. Using that $\tau_{2} / \tau_{1}=\xi_{1} / \xi_{2}$ depends only on $K$, we conclude the inequalities

$$
\begin{aligned}
\frac{1}{\sqrt{R_{c}\left(\xi_{1} \tau_{1}, \theta_{1}, t_{1}\right)+E}} & \geq \frac{\tilde{C}(K) \sqrt{\log \tau_{2}}}{\sqrt{\tau_{2}}} \\
& \geq \frac{\tilde{C}_{1}(K) \sqrt{\log \tau_{1}}}{\sqrt{\tau_{1}}}
\end{aligned}
$$

for $\tau_{1}$ sufficiently large, depending only on $K$. Estimate (4.13) yields the bound

$$
R_{c}\left(\xi_{1} \tau_{1}, \theta_{1}, t_{1}\right) \leq \frac{C(K) \tau_{1}}{\log \tau_{1}}
$$

finishing the proof of the Proposition.

Corollary 4.6. Under the assumptions of Theorem 1.2, we have

$$
\lim _{\tau \rightarrow \infty} \frac{1}{\tau} \int_{0}^{2 \pi}(\log \tilde{v})_{\xi \xi}(\xi, \theta, \tau) d \theta=0
$$

uniformly on compact subsets of $(T, \infty)$.

Proof. We begin by integrating (4.4) in $\theta$ which gives

$$
\int_{0}^{2 \pi}(\log \tilde{v})_{\xi \xi}(\xi, \theta, \tau) d \theta=-\int_{0}^{2 \pi} R_{c}(\xi \tau, \theta, t) \tilde{v}(\xi, \theta, \tau) d \theta, \quad \tau=\frac{1}{T-t} .
$$

Let $K \subset(T, \infty)$ compact. By the Aronson-Benilán inequality and Proposition 4.4

$$
-\frac{1}{t} \leq R_{c}(\xi \tau, \theta, t) \leq \frac{C(K) \tau}{\log \tau} .
$$

Since $\tilde{v} \leq C$ (by Lemma 4.1), we conclude

$$
\left|\frac{1}{\tau} \int_{0}^{2 \pi}(\log \tilde{v})_{\xi \xi}(\xi, \theta, \tau) d \theta\right| \leq \frac{C(K)}{\log \tau}
$$

from which the lemma directly follows.

We next introduce the new time variable

$$
s=\log \tau=-\log (T-t), \quad s \geq-\log T .
$$

To simplify the notation we still call $\tilde{v}(\xi, \theta, s)$ the solution $\tilde{v}$ in the new time scale. Then, it is easy to compute that $\tilde{v}(\xi, \theta, s)$ satisfies the equation

$$
\tilde{v}_{s}=e^{-s}(\log \tilde{v})_{\xi \xi}+e^{s}(\log \tilde{v})_{\theta \theta}+\xi \tilde{v}_{\xi}+2 \tilde{v} .
$$


For an increasing sequence of times $s_{k} \rightarrow \infty$, we let

$$
\tilde{v}_{k}(\xi, s)=\tilde{v}\left(\xi, s+s_{k}\right), \quad-\log T-s_{k}<s<\infty .
$$

Each $\tilde{v}_{k}$ satisfies the equation

$$
\left(\tilde{v}_{k}\right)_{s}=e^{-\left(s+s_{k}\right)}\left(\log \tilde{v}_{k}\right)_{\xi \xi}+e^{s+s_{k}}\left(\log \tilde{v}_{k}\right)_{\theta \theta}+\xi\left(\tilde{v}_{k}\right)_{\xi}+2 \tilde{v}_{k}
$$

and the area condition

$$
\int_{-\infty}^{\infty} \int_{0}^{2 \pi} \tilde{v}_{k}(\xi, \theta, s) d \theta d \xi=2
$$

Defining the functions

$$
W_{k}(\eta, s)=\int_{\eta}^{\infty} \int_{0}^{2 \pi} \tilde{v}_{k}(\xi, \eta, s) d \theta d \xi, \quad \eta \in(T, \infty),-\log T-s_{k}<s<\infty
$$

we have:

Lemma 4.7. Passing to a subsequence, $\left\{W_{k}\right\}$ converges uniformly on compact subsets of $\eta \in(T, \infty)$ to the time-independent steady state $2 T / \eta$. In addition, for any $p \geq 1$ and $\xi_{0} \in(T, \infty)$, the solution $\tilde{v}_{k}(\xi, \theta, s)$ of 4.16) converges in $L^{p}\left(\left[\xi_{0}, \infty\right) \times[0,2 \pi]\right)$ norm to $2 T / \xi^{2}$.

Proof. We first integrate (4.16) in $\theta$ and $\xi \in[\eta, \infty)$, for $\eta \in(T, \infty)$, to find that each $W_{k}$ satisfies the equation

$$
\left(W_{k}\right)_{s}=-\int_{\eta}^{\infty} \int_{0}^{2 \pi} \frac{\left(\log \tilde{v}_{k}\right)_{\xi \xi}}{\tau_{k}(s)} d \theta d \xi+\int_{\eta}^{\infty} \int_{0}^{2 \pi} \xi\left(\tilde{v}_{k}\right)_{\xi} d \theta d \xi+2 W_{k}
$$

with $\tau_{k}(s)=e^{-\left(s+s_{k}\right)}$. Integrating by parts the second term yields

$$
\begin{aligned}
\int_{\eta}^{\infty} \int_{0}^{2 \pi} \xi\left(\tilde{v}_{k}\right)_{\xi} d \theta d \xi & =-W_{k}(\eta, s)-\eta \int_{0}^{2 \pi} \tilde{v}_{k}(\eta, \theta, s) d \theta+\int_{0}^{2 \pi} \lim _{\xi \rightarrow \infty} \xi \tilde{v}_{k}(\xi, \theta, s) d \theta \\
& =-W_{k}(\eta, s)-\eta \int_{0}^{2 \pi} \tilde{v}_{k}(\eta, \theta, s) d \theta
\end{aligned}
$$

since due to our estimates on $\tilde{v}$ in Lemma 4.1 we have $\lim _{\xi \rightarrow \infty} \xi \tilde{v}_{k}(\xi, \theta, s)=0$, uniformly in $k$ and $\theta$. We conclude that

$$
\left(W_{k}\right)_{s}=-\int_{\eta}^{\infty} \int_{0}^{2 \pi} \frac{\left(\log \tilde{v}_{k}\right)_{\xi \xi}}{\tau_{k}(s)} d \theta d \xi+W_{k}+\eta\left(W_{k}\right)_{\eta}
$$

Let $K \subset(T, \infty)$ compact. Then, by 4.14

$$
\left|\int_{\eta}^{\infty} \int_{0}^{2 \pi} \frac{\left(\log \tilde{v}_{k}\right) \xi \xi}{\tau_{k}(s)} d \theta d \xi\right| \leq \frac{C(K)}{s+s_{k}}
$$


TYPE II EXTINCTION PROFILE OF MAXIMAL SOLUTIONS TO THE RICCI FLOW IN $\mathbb{R}^{2} 23$

Also, by Lemma 4.1 and Proposition 4.4 there exists a constant $C=C(K)$ for which the bounds

$$
\left|W_{k}(\eta, s)\right| \leq C, \quad\left|\left(W_{k}\right)_{s}(\eta, s)\right| \leq C, \quad\left|\left(W_{k}\right)_{\eta}(\eta, s)\right| \leq C
$$

hold, for $s \geq-\log T$. Hence, passing to a subsequence, $W_{k}(\eta, s)$ converges uniformly on compact subsets of $(T, \infty) \times \mathbb{R}$ to a solution $W$ of the equation

$$
W_{s}=\eta W_{\eta}+W=(\eta W)_{\eta} \quad \text { on }(T, \infty) \times \mathbb{R}
$$

with

$$
\lim _{\eta \rightarrow T} W(\eta, s)=2, \quad s \in \mathbb{R}
$$

and

$$
\lim _{\eta \rightarrow \infty} W(\eta, s)=0, \quad s \in \mathbb{R} .
$$

As in [12], one can show that $W$ is completely determined by its boundary values at $T$, and it is is the steady state

$$
W(\eta, s)=\frac{2 T}{\eta}, \quad \eta>T, s \in \mathbb{R} .
$$

To show the $L^{p}$ convergence, we first notice that by the comparison principle

$$
v(\zeta, t) \leq \frac{2 T}{\left(\zeta-\zeta_{0}\right)^{2}}, \quad \zeta \geq \zeta_{0}, 0<t<T
$$

for $\zeta_{0}=\log \rho$, with $\rho$ denoting the radius of the support of $u_{0}$. This yields the bound

$$
\tilde{v}_{k}(\xi, \theta, s) \leq \frac{2 T}{\left(\xi-\zeta_{0} / \tau_{k}(s)\right)^{2}}, \quad \xi \geq T .
$$

By the triangle inequality we have

$$
\begin{aligned}
\int_{\eta}^{\infty} \int_{0}^{2 \pi}\left|\frac{2 T}{\xi^{2}}-\tilde{v}_{k}\right| d \theta d \xi & \leq \int_{\eta}^{\infty} \int_{0}^{2 \pi}\left(\frac{2 T}{\left(\xi-\zeta_{0} / \tau_{k}(s)\right)^{2}}-\tilde{v}_{k}\right) d \theta d \xi \\
& +\int_{\eta}^{\infty} \int_{0}^{2 \pi}\left(\frac{2 T}{\left(\xi-\zeta_{0} / \tau_{k}(s)\right)^{2}}-\frac{2 T}{\xi^{2}}\right) d \theta d \xi
\end{aligned}
$$

where the second integral converges to zero, as $k \rightarrow \infty$, by the first part of the Lemma. It is easy to see that the third integral converges as well. This gives us the desired $L^{1}$ convergence, which immediately implies the $L^{p}$ convergence, since $\left|\tilde{v}_{k}(\xi, \theta, s)-2 T / \xi^{2}\right|$ is uniformly bounded on $\left[\xi_{0}, \infty\right) \times[0,2 \pi]$, for $\xi_{0} \geq T$ and $s \geq-\log T$.

Remark 4.8. The $L^{p}$ convergence in the previous Lemma, implies that there is a subsequence $k_{l}$ so that $\tilde{v}_{k_{l}}(\xi, \theta, s) \rightarrow 2 T / \xi^{2}$ pointwise, almost everywhere on $(T, \infty) \times[0,2 \pi]$. 
Set $\tau_{k}(s)=e^{s+s_{k}}$. Since

$$
\frac{(\log \tilde{v})_{\xi \xi}(\xi, \theta, \tau)}{\tau}+\tau(\log \tilde{v})_{\theta \theta}(\xi, \theta, \tau)=-\tau R_{c}(\xi \tau, \theta, \tau) \tilde{v}(\xi, \theta, t),
$$

we can rewrite 4.16) as

$$
\left(\tilde{v}_{k}\right)_{s}=-\frac{R_{c}}{\tau_{k}(s)} \tilde{v}_{k}+\xi\left(\tilde{v}_{k}\right)_{\xi}+2 \tilde{v}_{k} .
$$

We divide the equation by $\tilde{v}_{k}$ and integrate it in $\theta$. Denoting by $Z_{k}(\xi, s)=$ $\int_{0}^{2 \pi} \log \tilde{v}_{k}(\xi, \theta, s) d \theta$ we get

$$
\left(Z_{k}\right)_{s}=-\int_{0}^{2 \pi} \frac{R_{c}}{\tau_{k}(s)} d \theta+\xi\left(Z_{k}\right)_{\xi}+4 \pi
$$

Notice that by Proposition (4.4), we have

$$
\left|\frac{R_{c}}{\tau_{k}(s)}\right| \leq \frac{1}{\log \tau_{k}(s)}
$$

and that by Lemma 4.2

$$
\left|\left(Z_{k}\right)_{\xi}(\xi, s)\right|=\left|\int_{0}^{2 \pi}\left(\log \tilde{v}_{k}\right)_{\xi}(\xi, \theta, s) d \theta\right| \leq C(K)
$$

for $\xi \in K$, a compact subset of $(T, \infty)$ and $s \geq-s_{k}-\log T$. This also implies the bound

$$
\left|\left(Z_{k}\right)_{s}(\xi, s)\right| \leq C(K)
$$

Lemma 4.9. Passing to a subsequence, $\tilde{Z}_{k}(\xi, s)$ converges uniformly on compact subsets of $(T, \infty) \times \mathbb{R}$ to a solution $Z$ of the equation

$$
Z_{s}=\xi Z_{\xi}+4 \pi \quad \text { on }(T, \infty) \times \mathbb{R} .
$$

Proof. Let $E \subset(T, \infty) \times \mathbb{R}$ compact. Then according to the previous estimates, the sequence $\tilde{Z}_{k}$ is equicontinuous on $E$, hence passing to a subsequence it converges to a function $Z$. In addition, the estimate (4.20) readily implies that $Z$ is a solution of the first order equation (4.21).

Lemma 4.10. The function $Z$ is given by

$$
Z(\xi, s)=2 \pi \log \frac{2 T}{\xi^{2}}, \quad(\xi, s) \in(T, \infty) \times \mathbb{R} .
$$

Proof. Since $\int_{0}^{2 \pi} \log \tilde{v}_{k}(\xi, \theta, s) d \theta \rightarrow Z(\xi, s)$, uniformly in $\xi$ on compact subsets of $(T, \infty)$, then for any $A>0$ we have

$$
\int_{\eta}^{\eta+A} \int_{0}^{2 \pi} \log \tilde{v}_{k}(\xi, \theta, s) d \theta d \xi \rightarrow \int_{\eta}^{\eta+A} Z(\xi, s) d \xi
$$


TYPE II EXTINCTION PROFILE OF MAXIMAL SOLUTIONS TO THE RICCI FLOW IN $\mathbb{R}^{2} 25$

By Remark 4.8 and the dominated convergence theorem it follows that for every $A>0$ we have

$$
\int_{\eta}^{\eta+A} 2 \pi \log \frac{2 T}{\xi^{2}} d \xi=\int_{\eta}^{\eta+A} Z(\xi, s) d \xi
$$

implying that $Z$ is given by (4.22).

We are finally in position to conclude the proof of Theorem 1.2

Proof of Theorem 1.2. We begin by observing that by Lemma 4.1

$$
\tilde{v}_{k}(\xi, \theta, \tau) \rightarrow 0, \quad \text { as } \tau \rightarrow \infty
$$

uniformly on $\left(-\infty, \xi^{-}\right] \times[0,2 \pi]$, for any $-\infty<\xi^{-}<T$.

To show the convergence on the outer region, observe that by Lemma 4.9 and Lemma 4.10

$$
\int_{0}^{2 \pi} \log \tilde{v}_{k}(\xi, \theta, s) d \theta \rightarrow 2 \pi \log \frac{2 T}{\xi^{2}}
$$

uniformly on compact subsets of $(T, \infty) \times(-\infty, \infty)$. Set

$$
\underline{v}_{k}(\xi, s)=\min _{\theta \in[0,2 \pi]} \tilde{v}_{k}(\xi, \theta, s) \quad \text { and } \quad \bar{v}_{k}(\xi, s)=\max _{\theta \in[0,2 \pi]} \tilde{v}_{k}(\xi, \theta, s) .
$$

Let us recall that $u_{0} \subset B_{\rho}(0)$. By the monotonicity property of the solutions shown in Lemma 2.4 we have

$$
\begin{aligned}
2 \pi \log \underline{v}_{k}(\xi, s) & \leq \int_{0}^{2 \pi} \log \tilde{v}_{k}(\xi, \theta, s) d \theta \leq 2 \pi \log \bar{v}_{k}(\xi, s) \\
& \leq 2 \pi \log \frac{e^{2 \xi \tau_{k}(s)}}{\left(e^{\xi \tau_{k}(s)}-1\right)^{2}}+2 \pi \log \underline{v}_{k}\left(\xi+\frac{\log \left(1-\rho e^{-\xi \tau_{k}(s)}\right)}{\tau_{k}(s)}, s\right) \\
(4.24) & \leq 2 \pi \log \frac{e^{2 \xi \tau_{k}(s)}}{\left(e^{\xi \tau_{k}(s)}-\rho\right)^{2}}+\int_{0}^{2 \pi} \log \tilde{v}_{k}\left(\xi+\frac{\log \left(1-\rho e^{-\xi \tau_{k}(s)}\right)}{\tau_{k}(s)}, \theta, s\right) d \theta .
\end{aligned}
$$

Combining (4.23) and (4.24) yields that

$$
2 \pi \log \bar{v}_{k}(\xi, s) \rightarrow 2 \pi \log \frac{2 T}{\xi^{2}} \quad \text { and } \quad 2 \pi \log \underline{v}_{k}(\xi, s) \rightarrow 2 \pi \log \frac{2 T}{\xi^{2}}
$$

uniformly on compact subsets of $(T, \infty) \times \mathbb{R}$. Since

$$
\underline{v}_{k}(\xi, s) \leq \tilde{v}_{k}(\xi, \theta, s) \leq \bar{v}_{k}(\xi, s)
$$

the above readily implies that $\tilde{v}_{k}(\xi, \theta, s) \rightarrow 2 T / \xi^{2}$ uniformly on compact subsets of $(T, \infty) \times[0,2 \pi] \times(-\infty, \infty)$. Since the limit is independent of the sequence $s_{k} \rightarrow \infty$, we conclude that

$$
\tilde{v}(\xi, \theta, \tau) \rightarrow \frac{2 T}{\xi^{2}}, \quad \text { as } \tau \rightarrow \infty
$$


uniformly on compact subsets of $(T, \infty) \times[0,2 \pi]$, which finishes the proof of Theorem 1.2

\section{REFERENCES}

[1] Angenent, S. The zero set of a solution of a parabolic equation, J. Reine Angew. Math. 390 (1988), 79-96.

[2] Angenent, S., Knopf, D., Precise asymptotics of the Ricci flow neckpinch; preprint available on: www.ma.utexas.edu/ danknopf.

[3] Aronson, D.G., Bénilan P., Régularité des solutions de l'équation de milieux poreux dans $\mathbf{R}^{n}$, C.R. Acad. Sci. Paris, 288, 1979, pp 103-105.

[4] Aronson, D.G., Caffarelli,L.A., The initial trace of a solution of the porous medium equation, Transactions of the Amer. Math. Soc. 280 (1983), 351-366.

[5] Bertozzi, A.L., The mathematics of moving contact lines in thin liquid films, Notices Amer. Math. Soc. 45 (1998), no. 6, pp 689-697.

[6] Bertozzi, A.L., Pugh M., The lubrication approximation for thin viscous films: regularity and long-time behavior of weak solutions, Comm. Pure Appl. Math. 49 (1996), no. 2, pp 85-123.

[7] Cao, H.-D., Chow, B., Recent developments on the Ricci flow, Bull. Amer. Math. Soc. (N.S.) 36 (1999), no. 1, pp 59-74.

[8] Chow, B., The Ricci flow on the 2-sphere. J. Differential Geom. 33 (1991), no. 2, 325-334.

[9] Chow, B., On the entropy estimate for the Ricci flow on compact 2-orbifolds. J. Differential Geom. 33 (1991), no. 2, 597-600.

[10] de Gennes, P.G., Wetting: statics and dynamics, Reviews of Modern Physics, 57 No 3, 1985, pp 827-863.

[11] Daskalopoulos,P., del Pino M.A., On a Singular Diffusion Equation, Comm. in Analysis and Geometry, Vol. 3, 1995, pp 523-542.

[12] Daskalopoulos,P., del Pino M.A., Type II collapsing of maximal Solutions to the Ricci flow in $\mathbb{R}^{2}$, to appear in Ann. Inst. H. Poincar Anal. Non Linaire.

[13] Daskalopoulos, P., Hamilton, R., Geometric Estimates for the Logarithmic Fast Diffusion Equation, Comm. in Analysis and Geometry, 2004, to appear.

[14] Daskalopoulos, P., Sesum, N., Eternal solutions to the Ricci flow on $\mathbb{R}^{2}$, preprint.

[15] Esteban, J.R., Rodríguez, A., Vazquez, J.L., A nonlinear heat equation with singular diffusivity, Arch. Rational Mech. Analysis, 103, 1988, pp. 985-1039.

[16] Galaktionov,V.A., Peletier, L.A., Vazquez, J.L., Asymptotics of the fast-diffusion equation with critical exponent; Siam. J. Math. Anal., 31(1999), 1157-1174.

[17] Galaktionov, Victor A.; Vazquez, Juan Luis A stability technique for evolution partial differential equations. A dynamical systems approach. Progress in Nonlinear Differential Equations and their Applications, 56. Birkhauser Boston, Inc., Boston, MA, 2004.

[18] Hamilton, R., Yau, S-T, The Harnack estimate for the Ricci flow on a surface - Revisited, Asian J. Math, Vol 1, No 3, pp. 418-421.

[19] Hamilton, R., The Ricci flow on surfaces, Contemp. Math., 71, Amer. Math. Soc., Providence, RI, 1988, pp 237-262. 
TYPE II EXTINCTION PROFILE OF MAXIMAL SOLUTIONS TO THE RICCI FLOW IN $\mathbb{R}^{2} 27$

[20] Hamilton, R. The formation of singularities in the Ricci flow, Surveys in differential geometry, Vol. II pp 7-136, Internat. Press, Cambridge, MA, 1995.

[21] Hamilton, R., The Harnack estimate for the Ricci Flow, J. Differential Geometry 37 (1993) pp 225-243.

[22] Herrero, M. and Pierre, M., The Cauchy problem for $u_{t}=\Delta u^{m}$ when $0<m<1$, Trans. Amer. Math. Soc., 291, 1985, pp. 145-158.

[23] Hsu, S.-Y; Dynamics of solutions of a singular diffusion equation; Adv. Differential Equations 7 (2002), no. 1, 77-97.

[24] Hsu, Shu-Yu; Asymptotic profile of solutions of a singular diffusion equation as $t \rightarrow \infty$, Nonlinear Anal. 48 (2002), no. 6, Ser. A: Theory Methods, 781-790.

[25] Hsu, S.-Y; Large time behaviour of solutions of the Ricci flow equation on $R^{2}$, Pacific J. Math. 197 (2001), no. 1, 25-41.

[26] Hsu, S.-Y; Asymptotic behavior of solutions of the equation $u_{t}=\Delta \log u$ near the extinction time, Advances in Differential Equations, 8, No 2, (2003), pp 161-187.

[27] Hui, K.-M. Singular limit of solutions of the equation $u_{t}=\Delta\left(u^{m} / m\right)$ as $m \rightarrow 0$. Pacific J. Math. 187 (1999), no. 2, 297-316.

[28] King, J.R., Self-similar behavior for the equation of fast nonlinear diffusion, phil. Trans. R. Soc., London, A 343, (1993), pp 337-375.

[29] Rodriguez, A; Vazquez, J. L.; Esteban, J.R, The maximal solution of the logarithmic fast diffusion equation in two space dimensions, Adv. Differential Equations 2 (1997), no. 6, pp $867-894$.

[30] Wu, L.-F., A new result for the porous medium equation derived from the Ricci flow, Bull. Amer. Math. Soc., 28, 1993, pp 90-94.

[31] Wu, L.-F., The Ricci Flow on Complete $\mathbf{R}^{2}$, Communications in Analysis and Geometry, 1, 1993, pp 439-472.

Department of Mathematics, Columbia University, New York, USA

E-mail address: pdaskalo@math.columbia.edu

Department of Mathematics, Columbia University, New York, USA

E-mail address: natasas@math.columbia.edu 